\title{
Estudio de macronutrientes para la producción de PUFAs a partir de la microalga marina Isochrysis galbana.
}

\author{
Por J.L. García Sánchez, E. Molina Grima, F. García Camacho, J.A. Sánchez Pérez y D. López Alonso*. \\ Departamento de Ingeniería Química y *Departamento de Genética. \\ Facultad de Ciencias Experimentales. Universidad de Almería. 04071 Almería.
}

\section{RESUMEN}

Estudio de macronutrientes para la producción de PUFAs a partir de la microalga marina Isochrysis galbana.

Las microalgas marinas se presentan como una fuente alternativa en la producción de ácidos grasos poliinsaturados (PUFAs), frente a las fuentes convencionales, como son los aceites de pescado.

En este trabajo se presenta un estudio de macronutrientes para el medio de cultivo utilizado en el crecimiento de la microalga marina Isochrysis galbana, empleada con este objetivo.

La metodología de optimización aplicada ha sido la técnica de superficie de respuesta, siendo los factores estudiados el porcentaje de $\mathrm{CO}_{2}$ suplementario en la corriente de burbujeo, y las concentraciones de nitratos, fosfatos, citrato férrico y cloruro de manganeso en el medio de cultivo.

Los resultados de este trabajo ponen de manifiesto la existencia de limitaciones al crecimiento con el medio de partida y orientan a una segunda etapa de optimización, tomando como variables de diseño las relaciones entre los distintos componentes del medio de cultivo.

PALABRAS-CLAVE: Acido graso poliinsaturado - Isochrysis galbana Macronutriente (optimización) - Microalga marina.

\section{SUMMARY}

Macronutrient study for PUFAs production from the marine microalga Isochrysis galbana.

Marine microalgae are presented as alternative sources for polyunsaturated fatty acids production.

A macronutrient optimization method for culture media commonly used for Isochrysis galbana growth is discussed. This microalga has been reported as a good PUFA producer.

The optimization was conducted applying the response surface technique, parameters tested being nitrate, phosphate, ferric citrate and manganese chloride concentrations and the suplementary $\mathrm{CO}_{2}$ in the bubbling air.

The results show the evidence of growth limitation with the initial culture media and it is proposed a second optimization step considering as design variables the different ratios between media components.

KEY-WORDS: Isochrysis galbana - Macronutrient (optimization) Marine microalgae - Polyunsaturated fatty acid.

\section{INTRODUCCIÓN}

Los ácidos grasos poliinsaturados, (PUFAs) vienen siendo objeto de una gran variedad de estudios por su creciente interés en los últimos años. Este interés se debe a la relación que estos compuestos tienen en la nutrición de moluscos bivalvos y peces (1) (2) y con la salud humana. En este último caso, especialmente, por el carácter preventivo en patologías relacionadas con el sistema circulatorio (ácido eicosapentaenoico, EPA) (3) (4) (5) y en la formación y desarrollo del sis- tema nervioso central (ácido docosahexaenoico, DHA) (6) (7) (8).

En la actualidad la fuente comercial para estos ácidos grasos son los aceites de pescado, que presentan el problema de no tener una producción estable por las fluctuaciones en el volumen de las capturas y en la composición bioquímica de los peces de los que se extrae el aceite. Esta y otras razones han impulsado la búsqueda de fuentes alternativas entre las que se encuentran las microalgas (9) (10).

Los cultivos de microalgas están libres de contaminaciones a las que puedan estar sujetos los aceites de pescado, (metales pesados, pesticidas, etc) (11); presentan una composición química estable, ya que el cultivo se hace en condiciones controladas, y dan una producción continua a lo largo del año.

La posibilidad de desarrollar de modo comercial el cultivo de microalgas para la producción de PUFAs requiere establecer las condicones óptimas de cultivo.

En este sentido, el medio de cultivo es uno de los factores más utilizados para la modificación de la productividad en biomasa y composición bioquímica. Hay, por supuesto, otros factores ambientales, que se utilizan con el mismo propósito: temperatura, iluminación, caudales gaseosos, velocidad de dilución, etc. El medio de cultivo se diferencia de los anteriores en ser un multifactor, para una misma especie y sistema de cultivo determinados.

Así, podrían establecerse dos formas de operar: utilizar diferentes medios de cultivo con una formulación de nutrientes concreta y medir la respuesta para el estudio considerado (obtención de biomasa, polisacáridos, pigmentos, ácidos grasos, etc) o bien modificar la formulación de un único medio. Esta segunda opción es la más utilizada en los trabajos publicados (12) (13). De esta forma aparecen en bibliografía numerosos trabajos que estudian la influencia en el crecimiento de la concentración de uno o varios nutrientes, asociados generalmente con otro factor físico ambiental (14).

La metodología de optimización de un único medio de cultivo para maximizar el crecimiento y la producción de EPA y DHA puede acometerse por el método clásico, es decir, modificar una variable y fijar el resto en unos niveles concretos, lo que llevaría mucho tiempo de experimentación. Alternativamente, pueden aplicarse los métodos estadísticos, especialmente cuando el número de variables es elevado. 


\section{MATERIALES Y MÉTODOS}

La aplicación de la metodología de superficie de respuesta como alternativa a los métodos clásicos de optimización supone el desarrollo de tres etapas: plantear el diseño experimental, estimar los coeficientes en un modelo matemático y predecir la respuesta, comprobando la reproducibilidad del modelo (15). En este caso, como diseño experimental se aplican los diseños factoriales, cuya característica es evaluar la posible interacción entre los factores (16), evitando de esta forma la aparición de falsos óptimos (17).

En la optimización del medio de cultivo mediante la técnica de superficie de respuesta, deben haberse determinado previamente las variables que se tomarán como factores en el diseño experimental (18). En el caso que nos ocupa, partimos de una modificación del medio de Ukeles (19), cuya composición aparece en la Tabla I.

\section{Tabla I. Composición del medio a optimizar}

\begin{tabular}{|c|c|}
\hline Componente & Concentración en el medio \\
\hline $\mathrm{NaH}_{2} \mathrm{PO}_{4} 2 \mathrm{H}_{2} \mathrm{O}$ & $1,5610^{-2} \mathrm{~g} / \mathrm{l}$ \\
\hline $\mathrm{NaNO}_{3}$ & $1,710^{-2} \mathrm{~g} / \mathrm{l}$ \\
\hline $\mathrm{MnCl}_{2} 4 \mathrm{H}_{2} \mathrm{O}$ & $0,019810^{-2} \mathrm{~g} / \mathrm{l}$ \\
\hline $\mathrm{Na}_{2} \mathrm{MoO}_{4} 2 \mathrm{H}_{2} \mathrm{O}$ & $0,024210^{-2} \mathrm{~g} / \mathrm{l}$ \\
\hline $\mathrm{ZnCl}_{2}$ & $0,013610^{-2} \mathrm{~g} / \mathrm{l}$ \\
\hline $\mathrm{CoCl}_{2} 6 \mathrm{H}_{2} \mathrm{O}$ & $0,002510^{-2} \mathrm{~g} / \mathrm{l}$ \\
\hline $\mathrm{CuSO}_{4} 5 \mathrm{H}_{2} \mathrm{O}$ & $0,002510^{-2} \mathrm{~g} / \mathrm{l}$ \\
\hline citrato férrico & $0,4910^{-2} \mathrm{~g} / \mathrm{l}$ \\
\hline $\mathrm{Na}_{2}$-EDTA & $0,96810^{-2} \mathrm{~g} / \mathrm{l}$ \\
\hline tiamina & $35 \mu \mathrm{g} / \mathrm{l}$ \\
\hline biotina & $5 \mu g / 1$ \\
\hline cobalamina $\mathrm{B}_{12}$ & $3 \mu \mathrm{g} / /$ \\
\hline tampón Tris-HCl & $5 \mathrm{mM}$ \\
\hline
\end{tabular}

En experimentos previos realizados con esta microalga se comprobó un mayor crecimiento con $\mathrm{CO}_{2}$ suplementario, la no necesidad de tamponar el medio y se realizaron estudios de consumo de micronutrientes en cultivos batch. Los resultados mostraron que había un exceso de micronutrientes y que el citrato férrico y el cloruro de manganeso eran eliminados del medio.

Por otra parte, la influencia de la concentración de nitratos y fosfatos en el crecimiento y composición bioquímica de esta microalga está suficientemente probada (20) (21) (12) (13)

La técnica de superficie de respuesta es'un método de optimización secuencial e iterativo. En este trabajo se exponen los resultados de la primera fase que consiste en plantear el diseño para la recopilación de datos y analizar la superficie de respuesta de primera aproximación al óptimo (22).

De este modo, y puesto que se trata de optimizar los niveles de macronutrientes del medio de cultivo para la microalga Isochrysis galbana se optó por las variables para el diseño factorial que aparecen en la Tabla II. Éste es el diseño factorial incompleto de Box-Behnken con tres niveles y cinco factores agrupados en un solo bloque, dando lugar a 46 experimentos (23), Tabla III. Los nutrientes considerados como factores en este diseño fueron: concentración de nitratos, porcentaje de $\mathrm{CO}_{2}$ en la corriente gaseosa de burbujeo, concentración de citrato férrico, concentración de fosfatos y concentración de cloruro de manganeso.

Tabla II. Niveles de concentración de las variables

\begin{tabular}{|llll|}
\hline Variables & -1 & 0 & 1 \\
\hline$\% \mathrm{CO}_{2}$ & 0,03 & 1 & 2 \\
$\mathrm{NaNO}_{3}$ & $0,17 \mathrm{~g} / \mathrm{l}$ & $0,85 \mathrm{~g} / \mathrm{l}$ & $2,55 \mathrm{~g} / \mathrm{l}$ \\
$\mathrm{NaH}_{2} \mathrm{PO}_{4} 2 \mathrm{H}_{2} \mathrm{O}$ & $0,0156 \mathrm{~g} / \mathrm{l}$ & $0,078 \mathrm{~g} / \mathrm{l}$ & $0,234 \mathrm{~g} / \mathrm{l}$ \\
citrato férrico & $0,0049 \mathrm{~g} / \mathrm{l}$ & $0,0245 \mathrm{~g} / \mathrm{l}$ & $0,0735 \mathrm{~g} / \mathrm{l}$ \\
$\mathrm{MnCl}_{2} 4 \mathrm{H}_{2} \mathrm{O}$ & $0,198 \mathrm{mg} / \mathrm{l}$ & $0,990 \mathrm{mg} / \mathrm{l}$ & $2,97 \mathrm{mg} / \mathrm{l}$ \\
\hline
\end{tabular}

Como se puede apreciar en la Tabla III, este diseño permite la agrupación de los experimentos en tres series experimentales distintas, siendo dos de ellas el resultado de la aplicación de los diseños de Box-Behnken para tres factores (Tabla IV) (23). Esto permite el estudio independiente de dos series experimentales con tres factores y tres niveles.

De esta forma, en la primera serie experimental, $X_{1}$ corresponde al porcentaje de $\mathrm{CO}_{2}$ en el aire suministrado al cultivo, $\mathrm{X}_{2}$ es la concentración inicial de nitratos en el medio de cultivo en el momento de la inoculación y $\mathrm{X}_{3}$ es la concentración de fosfatos. El resto de los nutrientes se fijan en los valores que tienen en el medio de Ukeles (Tabla I), excepto para el citrato férrico y el cloruro de manganeso, que son $0,0245 \mathrm{~g} / \mathrm{l}$ y $0,99 \mathrm{mg} / \mathrm{l}$, respectivamente.

En la segunda serie experimental, $\mathrm{X}_{1}$ corresponde a la concentración de fosfatos en el medio al inicio del cultivo, $\mathrm{X}_{2}$ es la concentración de citrato férrico y $\mathrm{X}_{3}$ la de cloruro de manganeso. El resto de los nutrientes se fijan igual que la primera serie, excepto para la concentración de nitratos, que es $0,85 \mathrm{~g} / \mathrm{l}$ y el porcentaje de $\mathrm{CO}_{2}$ en el aire, que se fija en el $1 \%$. Por tanto, el diseño aplicado es una modificación del diseño de Box-Behnken para tres factores, (Tabla IV) con niveles no equiespaciados.

La microalga marina utilizada en este estudio ha sido un aislamiento (AL-II4) obtenido de un programa de mutación/selección sobre la especie Isochrysis galbana, referenciada como fuente de ácidos grasos poliinsaturados, principalmente EPA y DHA (24) (25), y con la que se viene trabajando en nuestro laboratorio desde 1989. Este aislamiento mostró una mejora considerable en el contenido en peso seco de EPA respecto al cultivo original (26).

Los experimentos se han realizado en vasos de cultivo cilíndricos y con camisa de termostatación, con un volumen útil de un litro, en una instalación provista para tal fin. Todos los cultivos se han realizado en discontinuo hasta alcanzar la fase estacionaria, a $20^{\circ} \mathrm{C}$ y $150 \mathrm{w} / \mathrm{m}^{2}$ de intensidad de iluminación continua (12). El caudal de aireación fue de $2 \mathrm{vv} . / \mathrm{min}$. (2 litros/min) en todos los casos, modifi- 
Tabla III. Diseño factorial de Box-Behnken para 5 factores

\begin{tabular}{|c|c|c|c|c|c|}
\hline \multirow[b]{2}{*}{ clave } & \multicolumn{5}{|c|}{ Factores } \\
\hline & $x_{1}$ & $x_{2}$ & $x_{3}$ & $x_{4}$ & $x_{5}$ \\
\hline$c$ & 0 & 0 & 0 & 0 & 0 \\
\hline s1 & 1 & 0 & 1 & 0 & 0 \\
\hline s1 & 1 & 0 & -1 & 0 & 0 \\
\hline$s 1$ & -1 & 0 & 1 & 0 & 0 \\
\hline s1 & -1 & 0 & -1 & 0 & 0 \\
\hline$c$ & 0 & 0 & 0 & 0 & 0 \\
\hline$s 1$ & 1 & 1 & 0 & 0 & 0 \\
\hline s1 & 1 & -1 & 0 & 0 & 0 \\
\hline s1 & -1 & 1 & 0 & 0 & 0 \\
\hline s1 & -1 & -1 & 0 & 0 & 0 \\
\hline$c$ & 0 & 0 & 0 & 0 & 0 \\
\hline s1 & 0 & 1 & 1 & 0 & 0 \\
\hline s1 & 0 & 1 & -1 & 0 & 0 \\
\hline s1 & 0 & -1 & 1 & 0 & 0 \\
\hline s1 & 0 & -1 & -1 & 0 & 0 \\
\hline$c$ & 0 & 0 & 0 & 0 & 0 \\
\hline s2 & 0 & 0 & 1 & 1 & 0 \\
\hline $\mathrm{s} 2$ & 0 & 0 & 1 & -1 & 0 \\
\hline s2 & 0 & 0 & -1 & 1 & 0 \\
\hline s2 & 0 & 0 & -1 & -1 & 0 \\
\hline$c$ & 0 & 0 & 0 & 0 & 0 \\
\hline s2 & 0 & 0 & 1 & 0 & 1 \\
\hline s2 & 0 & 0 & 1 & 0 & -1 \\
\hline s2 & 0 & 0 & -1 & 0 & 1 \\
\hline s2 & 0 & 0 & -1 & 0 & -1 \\
\hline$c$ & 0 & 0 & 0 & 0 & 0 \\
\hline s2 & 0 & 0 & 0 & 1 & 1 \\
\hline s2 & 0 & 0 & 0 & 1 & -1 \\
\hline s2 & 0 & 0 & 0 & -1 & 1 \\
\hline s2 & 0 & 0 & 0 & -1 & -1 \\
\hline$b$ & 0 & 1 & 0 & 1 & 0 \\
\hline$b$ & 0 & 1 & 0 & -1 & 0 \\
\hline b & 0 & -1 & 0 & 1 & 0 \\
\hline b & 0 & -1 & 0 & -1 & 0 \\
\hline b & 1 & 0 & 0 & 1 & 0 \\
\hline$b$ & 1 & 0 & 0 & -1 & 0 \\
\hline b & -1 & 0 & 0 & 1 & 0 \\
\hline b & -1 & 0 & 0 & -1 & 0 \\
\hline b & 1 & 0 & 0 & 0 & 1 \\
\hline$b$ & 1 & 0 & 0 & 0 & -1 \\
\hline$b$ & -1 & 0 & 0 & 0 & 1 \\
\hline$b$ & -1 & 0 & 0 & 0 & -1 \\
\hline$b$ & 0 & 1 & 0 & 0 & 1 \\
\hline$b$ & 0 & 1 & 0 & 0 & -1 \\
\hline$b$ & 0 & -1 & 0 & 0 & 1 \\
\hline$b$ & 0 & -1 & 0 & 0 & -1 \\
\hline
\end{tabular}

s1: primera serie experimental. $\left(\% \mathrm{CO}_{2}\right.$, nitratos y fosfatos como factores).

s2: segunda serie experimental. (fosfatos, citrato férrico y cloruro de manganeso como factores).

c: punto central del diseño.

b: combinación del diseño base (Box-Behnken de 5 factores).
Tabla IV. Diseño experimental de Box-Behnken para tres factores con tres puntos centrales

\begin{tabular}{|cccc|}
\hline Experimento & $\mathbf{X}_{1}$ & $\mathbf{X}_{2}$ & $\mathbf{X}_{3}$ \\
\hline 1 & 1 & 1 & 0 \\
2 & 1 & -1 & 0 \\
3 & -1 & 1 & 0 \\
4 & -1 & -1 & 0 \\
5 & 0 & 0 & 0 \\
6 & 1 & 0 & 1 \\
7 & 1 & 0 & -1 \\
8 & -1 & 0 & 1 \\
9 & -1 & 0 & -1 \\
10 & 0 & 0 & 0 \\
11 & 0 & 1 & 1 \\
12 & 0 & 1 & -1 \\
13 & 0 & -1 & 1 \\
14 & 0 & -1 & -1 \\
15 & 0 & 0 & 0 \\
\hline
\end{tabular}

cando el porcentaje de $\mathrm{CO}_{2}$ cuando fuera necesario, actuando sobre la válvula de $\mathrm{CO}_{2}$ previa a la corriente de mezcla.

En todos los experimentos se hizo un seguimiento diario de la concentración de biomasa y el $\mathrm{pH}$, dejando evolucionar los cultivos hasta fase estacionaria. Cuando el cultivo permanecía 24 horas en esta fase se determinaba el final del experimento. La concentración final a la que se cosechaba el cultivo se contrastaba frente a la densidad óptica a $530 \mathrm{~nm}$ por secado y pesada de un volumen conocido de cultivo.

La ecuación que relaciona la concentración en peso seco con la absorbancia a la longitud de onda citada fue: $\mathrm{C}(\mathrm{mg} / \mathrm{l})=\mathrm{DO}_{530^{\circ}} \cdot 232 ; \mathrm{r}^{2}=0,9987 ; \mathrm{n}=15(27)$

Los análisis de ácidos grasos se han realizado siguiendo el método de Lepage y Roy (28) (29) con las modificaciones recomendadas por García Sánchez y col. (30). Se ha utilizado una columna capilar de $30 \mathrm{~m}$ de longitud (SP2330, Supelco, Bellefonte, USA) con 0,25 mm de diámetro interno y $0,2 \mu \mathrm{m}$ de espesor de fase estacionaria. El flujo de gas portador $\left(\mathrm{N}_{2}\right)$ fue de $0,8 \mathrm{ml} / \mathrm{min}$ y se utilizó el sistema de división de flujo, con una relación de venteo 100:1. Los patrones para la identificación fueron PUFA-1, PUFA-2 y PUFA-3 suministrados por Supelco. Para la cuantificación se usó el método de patrón interno, siendo este el ácido nonadecanoico (19:0), añadido a la muestra antes de la metilación.

Como respuestas a estudiar en el diseño se han elegido: la concentración máxima de biomasa alcanzada por el cultivo (Cmax), velocidad específica de crecimiento $(\mu)$ y concentraciones de ácidos eicosapentaenóico ([EPA]) y docosahexaenoico ([DHA]) en el momento del cosechado. Las concentraciones de EPA y DHA, expresadas en $\mathrm{mg} / \mathrm{l}$, se han calculado multiplicando la concentración de biomasa (mg/l) en el momento de cosechado por el porcentaje en peso seco de dichos ácidos, obtenido del análisis por cromatografía gaseosa de dicha biomasa. La velocidad específica de crecimiento, expresada en $\mathrm{h}^{-1}$, se obtiene de la pendiente de la fase exponencial en la representación gráfica de $\mathrm{Ln}(\mathrm{C} / \mathrm{Co})$ vs tiempo de cultivo. 
Los resultados obtenidos para las respuestas citadas anteriormente se han sometido a un análisis de regresión múltiple, con el correspondiente análisis multifactorial de la varianza.

Todos los cálculos, tablas de resultados y gráficos se han obtenido con el paquete estadístico STATGRAPHICS versión 5 .

\section{RESULTADOS Y DISCUSIÓN}

En la Tabla $V$ se muestran los datos experimentales y en las Tablas VI a $X$ los resultados del análisis estadístico para las respuestas que se describieron en el apartado de metodología y diseño experimental. Así mismo se incluyen los coeficientes de regresión para el ajuste polinominal de segundo orden: es decir, los parámetros para la función superficie de respuesta. Dichos coeficientes aparecen con la codificación para el nivel de significación estadístico de la regresión.

El modelo empírico al que se ajustan los resultados es el siguiente:

$$
\begin{aligned}
Y= & B_{0}+B_{1} X_{1}+B_{2} X_{2}+B_{3} X_{3} \\
& +B_{12} X_{1} X_{2}+B_{13} X_{1} X_{3}+B_{23} X_{2} X_{3} \\
& +B_{11} X_{1} X_{1}+B_{22} X_{2} X_{2}+B_{33} X_{3} X_{3}
\end{aligned}
$$

Donde $Y$ es la superficie de respuesta considerada en cada caso, $X_{1}, X_{2}$ y $X_{3}$ son las variables que hemos modificado en la experimentación, es decir, los factores cuya influencia se pretende determinar y Bij son los parámetros del modelo (coeficientes de regresión de las Tablas VI a X).

En cada una de las tablas anteriores y en la columna $P$ aparece el nivel de significación estadística del ANOVA. También se indica en las mismas y en la columna Factores, aquellas proyecciones en tres dimen- siones de la superficie de respuesta considerada en cada caso, que presenten un nivel de confianza superior al $95 \%$. En la figura correspondiente se indica el valor al que se fija el tercer factor, ya que es imposible visualizar simultáneamente el espacio de los factores y la respuesta, que constituyen un espacio tetradimensional. Hay por tanto, tres posibles proyecciones para cada superficie de respuesta.

Las respuestas para la primera serie experimental, donde se analizan como factores las concentraciones de nitratos, fosfatos y el porcentaje de dióxido de carbono en la corriente de burbujeo, tienen bajos coeficientes de determinación $\left(r^{2}\right)$ para las respuestas Cmax: 0,6031 , [EPA]: 0,6389 y [DHA]: 0,4974, (datos no presentados), por lo que las superficies de respuesta de este estudio son poco fiables y deben desestimarse con fines predictivos.

Respecto a la velocidad específica de crecimiento $(\mu)$, que es la respuesta con mejor ajuste para esta serie, con un coeficiente de determinación de 0,8415 (Tabla VI), estaría limitada a las mínimas concentraciones de nitratos y fosfatos ensayadas simultáneamente, e inhibida a las mayores concentraciones en el mismo caso para el intervalo experimental considerado (figura 1). Es destacable el valor de la constante de la regresión para esta respuesta $\left(B_{0}\right), 0,027$ y su nivel de significación estadístico. Esto nos indica que, en la realización de la primera serie experimental, podría haber existido alguna limitación, que ha enmascarado la influencia de los factores y ha dado lugar a un mayor error experimental, ya que este valor debe tender a la velocidad específica de crecimiento máxima para el microorganismo estudiado, $0,032 \mathrm{~h}^{-1}$, cuanto mayor sea el coeficiente de

\begin{tabular}{|c|c|c|c|c|c|c|c|}
\hline \multicolumn{3}{|c|}{ Factores } & \multirow{2}{*}{$\begin{array}{l}\text { Serie1 } \\
\mu\left(h^{-1}\right)\end{array}$} & \multicolumn{4}{|c|}{ Serie2 } \\
\hline$x_{1}$ & $x_{2}$ & $x_{3}$ & & $\begin{array}{l}\text { Cmax } \\
(\mathrm{mg} / \mathrm{l})\end{array}$ & $\mu\left(h^{-1}\right)$ & $\begin{array}{c}\text { EPA } \\
(\mathrm{mg} / \mathrm{l})\end{array}$ & $\begin{array}{c}\text { DHA } \\
(\mathrm{mg} / \mathrm{l})\end{array}$ \\
\hline 0 & 1 & -1 & 0,0302 & 1418 & 0,0341 & 51,39 & 24,42 \\
\hline-1 & -1 & 0 & 0,0281 & 3254 & 0,0347 & 205,28 & 78,63 \\
\hline 1 & 0 & 1 & 0,0315 & 344 & 0,0247 & 4,17 & 2,56 \\
\hline-1 & 1 & 0 & 0,0304 & 1013 & 0,0232 & 43,62 & 21,41 \\
\hline 0 & 0 & 0 & 0,0319 & 995 & 0,0319 & 27,07 & 13,78 \\
\hline 0 & -1 & -1 & 0,0283 & 690 & 0,0338 & 15,14 & 7,67 \\
\hline 0 & 0 & 0 & 0,0299 & 1200 & 0,0299 & 45,94 & 22,58 \\
\hline 0 & -1 & 1 & 0,0316 & 1476 & 0,0319 & 59,00 & 26,68 \\
\hline 1 & 0 & -1 & 0,0284 & 729 & 0,0313 & 18,13 & 8,02 \\
\hline 1 & -1 & 0 & 0,0276 & 1195 & 0,0339 & 43,50 & 17,79 \\
\hline 0 & 1 & 1 & 0,0276 & 1342 & 0,0267 & 40,77 & 17,83 \\
\hline 0 & 0 & 0 & 0,0297 & 1476 & 0,0297 & 44,71 & 18,63 \\
\hline 1 & 1 & 0 & 0,0318 & 2642 & 0,0261 & 315,09 & 97,92 \\
\hline-1 & 0 & 1 & 0,0314 & 3000 & 0,0302 & 147,23 & 50,37 \\
\hline-1 & 0 & -1 & 0,0318 & 2893 & 0,0256 & 161,01 & 60,61 \\
\hline
\end{tabular}
determinación (12).

Tabla V. Resultados experimentales

Serie 1: $X_{1}$ porcentaje de $\mathrm{CO}_{2}, X_{2}$ concentración de nitratos, $X_{3}$ concentración de fosfatos.

Serie 2: $X_{1}$ concentración de fosfatos, $X_{2}$ concentración de citrato férrico, $X_{3}$ concentración de cloruro de manganeso. 


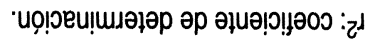

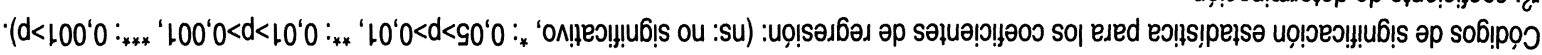

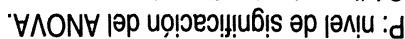

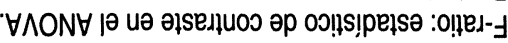
рецәq!| әр sopeג6 sopespeno әp euns :OSS

\begin{tabular}{|c|c|c|c|c|c|c|}
\hline & & & & & $9 Z 18^{\prime} 0$ & $\tau^{d}$ \\
\hline & & & & $\nabla t$ & ZLZGLLLL & |Eło| \\
\hline & & & & $\mathfrak{G}$ & Et९SOZZ & 10112 \\
\hline $\mathrm{su}_{1} \mathrm{OL} \mathrm{L}^{\prime} \mathrm{g}-$ & ${ }^{\varepsilon \varepsilon_{g}}$ & $988^{\prime} 0$ & $90^{\prime} 0$ & $\downarrow$ & †9เレ乙 & $\varepsilon^{\varepsilon} \varepsilon_{X}$ \\
\hline $\mathrm{su}_{\perp-0} \varepsilon^{\prime} \vdash$ & $z_{g}$ & SEL'0 & $\varepsilon L^{\prime} 0$ & 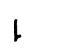 & $6 \angle 189$ & ${ }^{2} x^{2} x$ \\
\hline$* 2-010$ 0' 6 & $\mathrm{H}_{\mathrm{g}}$ & $8+0 \cdot 0$ & $0<' 9$ & 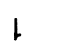 & $100 \angle 962$ & ${ }^{\prime} x t^{\prime} x$ \\
\hline $\mathrm{Su}_{L-0 L} L^{\prime} \mathrm{G}-$ & $\varepsilon^{\varepsilon} g$ & $\varepsilon \varepsilon \sigma^{\prime} 0$ & $10^{\prime} 0$ & $t$ & $\varepsilon \not t \varepsilon$ & $\varepsilon^{2} x$ \\
\hline su $8^{\prime} \vdash-$ & ${ }^{\varepsilon l_{g}}$ & ZLt'0 & $\varepsilon 8^{\prime} 0$ & $b$ & $0 \varepsilon \vdash \angle 9 \varepsilon$ & $\varepsilon^{\varepsilon}{ }^{\prime} x$ \\
\hline$* 1-01 \varepsilon^{\prime} Z$ & $\mathrm{I}_{\mathrm{g}}$ & $\varepsilon \varepsilon 0^{\prime} 0$ & $1 t^{\prime} 8$ & $b$ & $89 Z 60 \angle \varepsilon$ & $\left.{ }^{(t 6} 6 ! y\right){ }^{2} x+x$ \\
\hline$s u_{Z} O L L^{\prime} \forall-$ & $\varepsilon_{g}$ & $9966^{\prime} 0$ & $00^{\prime} 0$ & t & 796 & ${ }^{\varepsilon} x:\left[u w^{2} \mid \supset\right]$ \\
\hline $\mathrm{su}_{1}$ Ol t' & ${ }^{2} g$ & $299^{\prime} 0$ & $0 t^{\prime} 0$ & 1 & Z6IGLL & ${ }^{2} x:$ [0ग!1 \\
\hline * & $t_{g}$ & $\varepsilon 60^{\prime} 0$ & $8 Z^{\prime} t$ & $t$ & $1 t \angle 9881$ & ${ }^{\prime} \mathrm{X}:[$ solejsol] \\
\hline * $\varepsilon 0 / \varsigma^{\prime} \varepsilon$ & ${ }^{0} g$ & & & & & \\
\hline \multicolumn{2}{|c|}{ 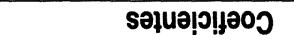 } & d & O!̣ed-d & \pm 0 & OSS & saloloed \\
\hline
\end{tabular}

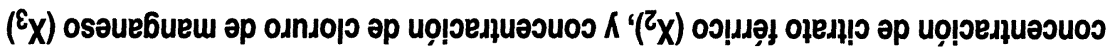

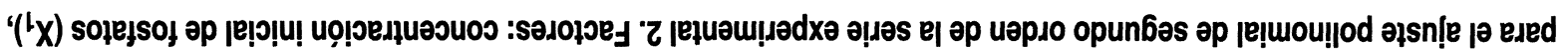

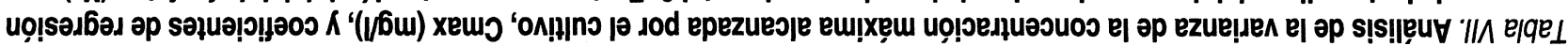

\begin{tabular}{|c|c|c|c|c|c|c|}
\hline & & & & & SLt88 0 & لـ \\
\hline & & & & tt & $\begin{array}{l}68 \uparrow £ \\
\varepsilon \varsigma \varsigma\end{array}$ & $\begin{array}{l}|\mathrm{E}| 0 \mid \\
101 \mathrm{t} \mid\end{array}$ \\
\hline $\mathrm{su}_{8}-0 \mathrm{Ol} \mathrm{L}^{\prime} \mathrm{C}$ & $\varepsilon \varepsilon_{g}$ & $\varepsilon 99^{\prime} 0$ & $2 Z^{\prime} 0$ & $b$ & $\downarrow z$ & $\varepsilon^{8}{ }^{8} x$ \\
\hline$* 6.01+t^{6}-1-$ & $2 \sigma_{g}$ & $9 \varepsilon 0^{\prime} 0$ & $20 ' 8$ & b & 988 & ${ }^{2} x^{2} x$ \\
\hline $\mathrm{Su}_{t}-\mathrm{Ol} \mathrm{O}^{\prime} \varepsilon^{-}$ & $H_{g}$ & $66 S^{\prime} 0$ & ट $\varepsilon^{\prime} 0$ & 1 & $9 \varepsilon$ & ${ }^{\prime} x^{\prime} x$ \\
\hline$=8.01 Z^{\prime} \mathrm{L}^{-}$ & $\varepsilon_{g}$ & $120^{\prime} 0$ & $66^{\prime} \mathrm{OH}$ & 1 & SLટL & ${ }^{8} x^{2} x$ \\
\hline $\mathrm{su}_{9-0 \mathrm{~L}} L^{\prime} \mathrm{g}$ & $\varepsilon l_{g}$ & $11 Z^{\prime} 0$ & so'z & b & $\angle Z Z$ & ${ }^{\varepsilon} x^{\prime} x$ \\
\hline $\mathrm{su}_{8}-\mathrm{Ol} \mathrm{L}^{\prime} \mathrm{E}-$ & 2tg & s\&6 0 & $10^{\prime} 0$ & 1 & 1 & $(1,6 y) x^{2} x^{2} x$ \\
\hline su $_{9.01} \sigma^{\prime} \varepsilon$ & $\varepsilon_{g}$ & ¿६8'0 & $90^{\prime} 0$ & 1 & 9 & EX $X:[$ solejejsol] \\
\hline$* 9.01$ E's & zg & ISL'O & टl'0 & b & $\varepsilon$ & ${ }^{2} x:[$ [solenentu] \\
\hline $\mathrm{su}_{t}-0 \mathrm{l} \mathrm{O}^{\prime} \mathrm{g}-$ & $\mathrm{tg}$ & $8 \angle Z^{\prime} 0$ & $8 t^{\prime} t$ & 1 & $t 9 t$ & ${ }^{\prime} x:{ }^{2} 00 \%$ \\
\hline$* * z-01<' z$ & og & & & & & \\
\hline \multicolumn{2}{|c|}{ sә|цә|ฺ|ןرə0ગ } & $d$ & oḷed-g & \pm 0 & OSS & SOגOloE] \\
\hline
\end{tabular}

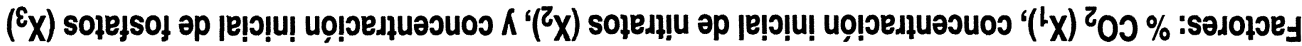

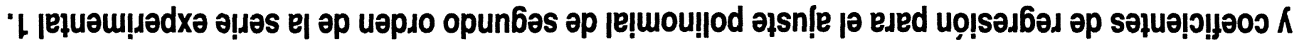

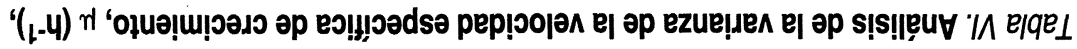


Tabla VIII. Análisis de la varianza de la velocidad específica de crecimiento, $\mu\left(\mathbf{h}^{-1}\right)$, y coeficientes de regresión para el ajuste polinomial de segundo orden de la serie experimental 2. Factores: concentración inicial de fosfatos $\left(X_{1}\right)$, concentración de citrato férrico $\left(X_{2}\right)$, y concentración de cloruro de manganeso $\left(X_{3}\right)$

\begin{tabular}{|c|c|c|c|c|c|c|}
\hline \multirow[t]{2}{*}{ Factores } & \multirow[t]{2}{*}{ SSQ } & \multirow[t]{2}{*}{ DF } & \multirow[t]{2}{*}{ F-ratio } & \multirow[t]{2}{*}{$\mathbf{P}$} & \multicolumn{2}{|c|}{ Coeficientes } \\
\hline & & & & & $\mathrm{B}_{0}$ & $3,110^{-2 * * *}$ \\
\hline [fosfatos]: $\mathrm{X}_{1}$ & 25 & 1 & 0,02 & 0,884 & $\mathrm{~B}_{1}$ & $8,710^{-5} \mathrm{~ns}$ \\
\hline [citrato férrico]: $X_{2}$ & 7217 & 1 & 6,91 & 0,046 & $\mathrm{~B}_{2}$ & $-2,510^{-4} \mathrm{~ns}$ \\
\hline$\left[\mathrm{Cl}_{2} \mathrm{Mn}\right]_{\mathrm{j}}: \mathrm{X}_{3}$ & 3019 & 1 & 2,89 & 0,149 & $\mathrm{~B}_{3}$ & $3,210^{-4} \mathrm{~ns}$ \\
\hline$x_{1} x_{2}$ & 1 & 1 & 0,00 & 0,983 & $\mathrm{~B}_{12}$ & $-9,010^{-9} \mathrm{~ns}$ \\
\hline$x_{1} x_{3}$ & 2267 & 1 & 2,17 & 0,200 & $\mathrm{~B}_{13}$ & $-1,510^{-5} \mathrm{~ns}$ \\
\hline$X_{2} X_{3 \text { (fig 5) }}$ & 480 & 1 & 0,46 & 0,534 & $\mathrm{~B}_{23}$ & $-2,110^{-5} \mathrm{~ns}$ \\
\hline$x_{1} x_{1}$ & 2228 & 1 & 2,13 & 0,204 & $\mathrm{~B}_{11}$ & $-2,610^{-7} \mathrm{~ns}$ \\
\hline$x_{2} x_{2}$ & 1980 & 1 & 1,90 & 0,227 & $\mathrm{~B}_{22}$ & $2,510^{-6} \mathrm{~ns}$ \\
\hline$x_{3} x_{3}$ & 55 & 1 & 0,05 & 0,830 & $\mathrm{~B}_{33}$ & $2,510^{-4} \mathrm{~ns}$ \\
\hline error & 5222 & 5 & & & & \\
\hline total & 19704 & 14 & & & & \\
\hline$r^{2}$ & 0,7349 & & & & & \\
\hline
\end{tabular}

Tabla IX. Análisis de la varianza de la concentración de EPA (mg/l) y coeficientes de regresión para el ajuste polinomial de segundo orden de la serie experimental 2. Factores: concentración inicial de fosfatos $\left(X_{1}\right)$, concentración de citrato férrico $\left(X_{2}\right)$, y concentración de cloruro de manganeso $\left(X_{3}\right)$

\begin{tabular}{|c|c|c|c|c|c|c|}
\hline \multirow[t]{2}{*}{ Factores } & \multirow[t]{2}{*}{ SSQ } & \multirow[t]{2}{*}{ DF } & \multirow[t]{2}{*}{ F-ratio } & \multirow[t]{2}{*}{$\mathbf{P}$} & \multicolumn{2}{|c|}{ Coeficientes } \\
\hline & & & & & $\mathrm{B}_{0}$ & $2,410^{2 \star \star}$ \\
\hline [fosfatos]: $\mathrm{X}_{1}$ & 3 & 1 & 0,00 & 0,966 & $\mathrm{~B}_{1}$ & $-3,2^{* *}$ \\
\hline [citrato férrico]:: $\mathrm{X}_{2}$ & 10360 & 1 & 7,29 & 0,042 & $\mathrm{~B}_{2}$ & $-4,7 \mathrm{~ns}$ \\
\hline$\left[\mathrm{Cl}_{2} \mathrm{Mn}_{\mathrm{i}}: \mathrm{X}_{3}\right.$ & 64 & 1 & 0,05 & 0,841 & $\mathrm{~B}_{3}$ & $5,910^{1} \mathrm{~ns}$ \\
\hline$X_{1} X_{2 \text { (fig 2) }}$ & 58837 & 1 & 41,38 & 0,001 & $\mathrm{~B}_{12}$ & $2,310^{-2 * * *}$ \\
\hline$x_{1} x_{3}$ & 718 & 1 & 0,51 & 0,516 & $\mathrm{~B}_{13}$ & $-8,010^{-2} \mathrm{~ns}$ \\
\hline$x_{2} x_{3}$ & 124 & 1 & 0,09 & 0,782 & $\mathrm{~B}_{23}$ & $-1,110^{-1} \mathrm{~ns}$ \\
\hline$x_{1} x_{1}$ & 24913 & 1 & 17,52 & 0,008 & $\mathrm{~B}_{11}$ & $8,810^{-3 * *}$ \\
\hline$x_{2} x_{2}$ & 2613 & 1 & 1,84 & 0,233 & $B_{22}$ & $2,810^{-2} \mathrm{~ns}$ \\
\hline$x_{3} x_{3}$ & 1858 & 1 & 1,31 & 0,304 & $\mathrm{~B}_{33}$ & $-1,510^{1} \mathrm{~ns}$ \\
\hline error & 7109 & 5 & & & & \\
\hline total & 106462 & 14 & & & & \\
\hline $\mathrm{r}^{2}$ & 0,9332 & & & & & \\
\hline
\end{tabular}

SSQ: Suma de cuadrados.

DF: grados de libertad.

F-ratio: estadístico de contraste en el ANOVA.

P: nivel de significación del ANOVA.

Códigos de significación estadística para los coeficientes de regresión: (ns: no significativo, ${ }^{*}: 0,05>p>0,01,{ }^{* *}: 0,01>p>0,001,{ }^{* \star *}: 0,001>p$ ).

$r^{2}$ : coeficiente de determinación. 
Tabla X. Análisis de la varianza de la concentración de DHA (mg/l) y coeficientes de regresión para el ajuste polinomial de segundo orden de la serie experimental 2. Factores: concentración inicial de fosfatos $\left(X_{1}\right)$, concentración de citrato férrico $\left(X_{2}\right)$, y concentración de cloruro de manganeso $\left(X_{3}\right)$

\begin{tabular}{|c|c|c|c|c|c|c|}
\hline \multirow[t]{2}{*}{ Factores } & \multirow[t]{2}{*}{ SSQ } & \multirow[t]{2}{*}{ DF } & \multirow[t]{2}{*}{ F-ratio } & \multirow[t]{2}{*}{$\mathbf{P}$} & \multicolumn{2}{|c|}{ Coeficientes } \\
\hline & & & & & $\mathrm{B}_{0}$ & $8,810^{1 * *}$ \\
\hline [fosfatos];: $\mathrm{X}_{1}$ & 100 & 1 & 0,57 & 0,493 & $B_{1}$ & $-1,1^{\star \star}$ \\
\hline [citrato férrico];: $X_{2}$ & 788 & 1 & 4,42 & 0,089 & $\mathrm{~B}_{2}$ & $-1,6 \mathrm{~ns}$ \\
\hline$\left[\mathrm{Cl}_{2} \mathrm{Mn}\right]_{\mathrm{j}}: \mathrm{X}_{3}$ & 16 & 1 & 0,09 & 0,774 & $\mathrm{~B}_{3}$ & $1,910^{1} \mathrm{~ns}$ \\
\hline$X_{1} X_{2 \text { (fig 3) }}$ & 5568 & 1 & 31,24 & 0,002 & $\mathrm{~B}_{12}$ & $9,010^{-2}$ ** \\
\hline$x_{1} x_{3}$ & 42 & 1 & 0,24 & 0,650 & $\mathrm{~B}_{13}$ & $-1,910^{-2} \mathrm{~ns}$ \\
\hline$x_{2} x_{3}$ & 26 & 1 & 0,15 & 0,721 & $\mathrm{~B}_{23}$ & $-4,810^{-2} \mathrm{~ns}$ \\
\hline$x_{1} x_{1}$ & 2603 & 1 & 14,61 & 0,012 & $\mathrm{~B}_{11}$ & $3,010^{-2}$ * \\
\hline$x_{2} x_{2}$ & 355 & 1 & 2,00 & 0,216 & $\mathrm{~B}_{22}$ & $1,110^{-2} \mathrm{~ns}$ \\
\hline$x_{3} x_{3}$ & 217 & 1 & 1,22 & 0,319 & $\mathrm{~B}_{33}$ & $-5,1 \mathrm{~ns}$ \\
\hline error & 891 & 5 & & & & \\
\hline total & 10900 & 14 & & & & \\
\hline$r^{2}$ & 0,9182 & & & & & \\
\hline
\end{tabular}

SSQ: Suma de cuadrados.

DF: grados de libertad.

F-ratio: estadístico de contraste en el ANOVA.

$P$ : nivel de significación del ANOVA.

Códigos de significación estadística para los coeficientes de regresión: (ns: no significativo, ${ }^{*}: 0,05>p>0,01,{ }^{* \star}: 0,01>p>0,001,{ }^{\star \star \star}: 0,001>p$ ).

$r^{2}$ : coeficiente de determinación.

Respecto de la segunda serie experimental, las figuras 2 y 3 , correspondientes a las superficies de respuesta para las concentraciones de EPA y DHA, respectivamente, en función de las concentraciones de fosfatos y citrato férrico, son practicamente idénticas. Esto es coherente ya que se trata de dos ácidos grasos de una misma ruta metabólica, en la que uno (EPA) es sustrato para la formación del otro (DHA). Por consiguiente, es lógico que respondan de modo similar a los cambios del medio.

En estas figuras se muestra la importancia de una combinación de nutrientes: como se puede observar en estas superficies de respuesta, en ausencia de citrato férrico, el aumento de la concentración de fosfatos en el medio inhibe la formación de EPA y DHA. El mismo comportamiento se observa para los mismos factores, en la respuesta concentración máxima alcanzada por el cultivo (figura 4), incluso de forma más acusada. Sin embargo, a las concentraciones de hierro más altas ensayadas, un aumento de la concentración de fosfatos produce un aumento en la respuesta para ambos ácidos grasos.

Este hecho pone de manifiesto la importancia de los medios equilibrados en su composición de nutrientes y aparece otro factor como crítico, en este caso la relación fosfatos/hierro (31), que es la misma en los dos puntos de máxima respuesta de las figuras 2 y 3 . También apreciamos en estas figuras que no se alcanza el límite máximo

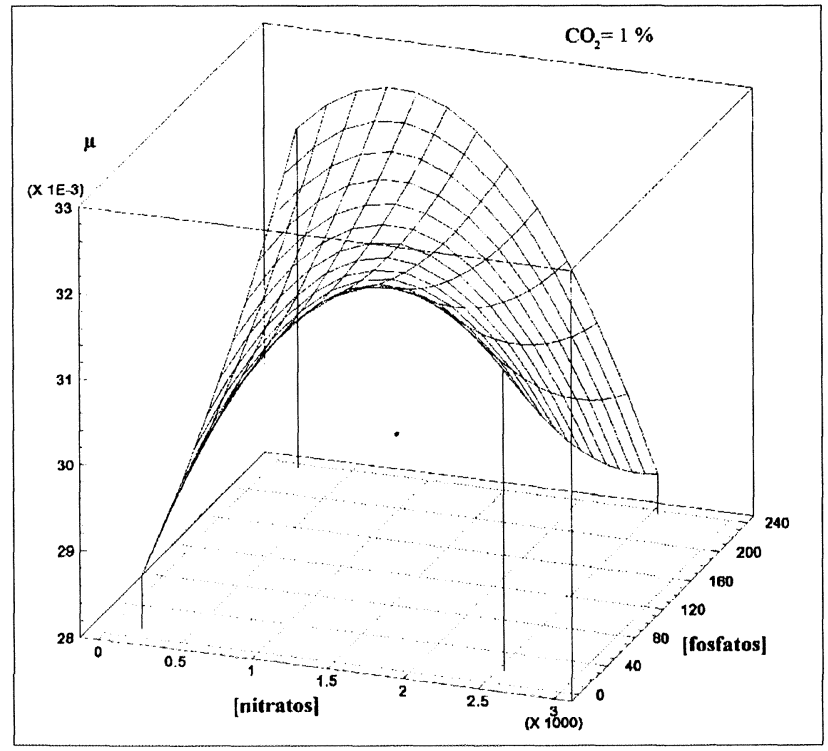

Figura 1

Superficie de respuesta estimada para la velocidad específica de crecimiento $(\mu)\left(h^{-1}\right)$ vs concentraciones de nitratos y fosfatos en el medio, en la primera serie experimental. El porcentaje de $\mathrm{CO}_{2}$ se fija al $1 \%$. Las unidades de los factores se expresan en $\mathrm{mg} / \mathrm{l}$. 
para el intervalo experimental considerado. Por tanto, se debería continuar el estudio en la dirección del aumento de fosfatos e hierro simultáneamente, manteniendo la relación crítica que hemos apuntado.

Referente a la otra respuesta considerada, velocidad específica de crecimiento $(\mu)$, y sin pretender ningún estudio cinético, la superficie de respuesta indica un hecho

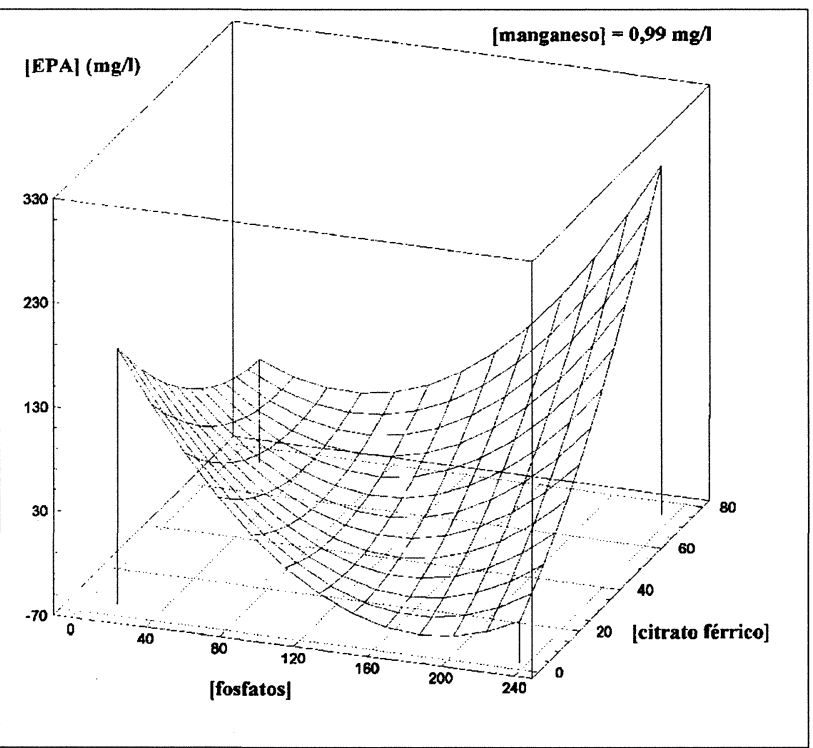

Figura 2

Superficie de respuesta estimada para la concentración de EPA (mg/l) en el momento del cosechado $v s$ concentraciones de citrato férrico y fosfatos en el medio, para la segunda serie experimental. La concentración de cloruro de manganeso se fija en $0,99 \mathrm{mg} / \mathrm{l}$

Las unidades de los factores se expresan en $\mathrm{mg} / \mathrm{l}$.

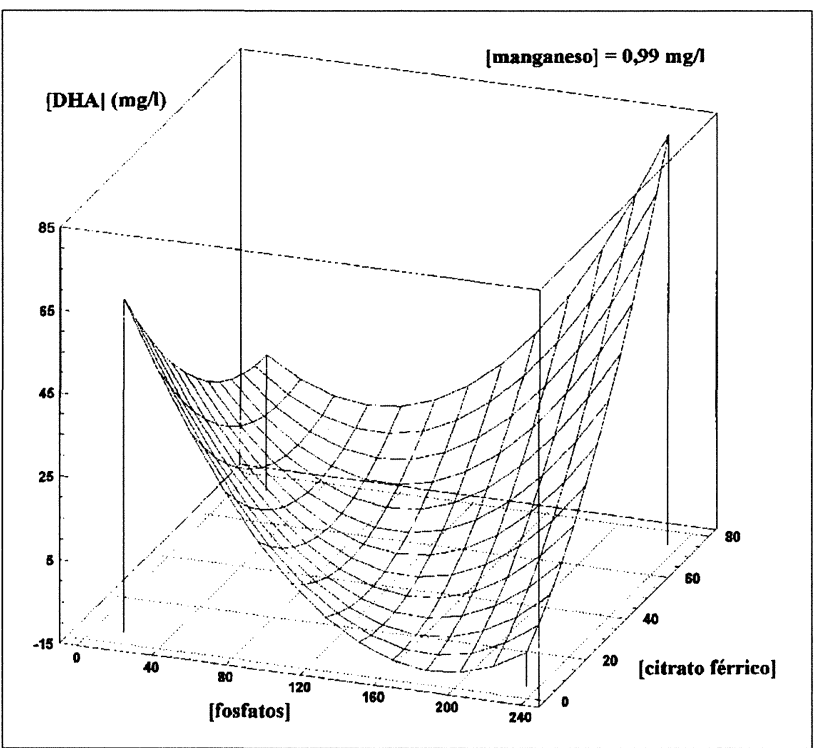

Figura 3

Superficie de respuesta estimada para la concentración de DHA (mg/l) en el momento del cosechado vs concentraciones de citrato férrico y fosfatos en el medio, para la segunda serie experimental. La concentración de cloruro de manganeso se fija en $0,99 \mathrm{mg} /$.

Las unidades de los factores se expresan en $\mathrm{mg} / \mathrm{l}$. muy claro: el efecto de la concentración de cloruro de manganeso es nulo o inhibidor a concentraciones de citrato férrico crecientes (figura 5).

La velocidad específica de crecimiento $(\mu)$, presenta otro hecho interesante: el valor de $\mathrm{B}_{0}, 0,031$, en el modelo de regresión coincide prácticamente con el de

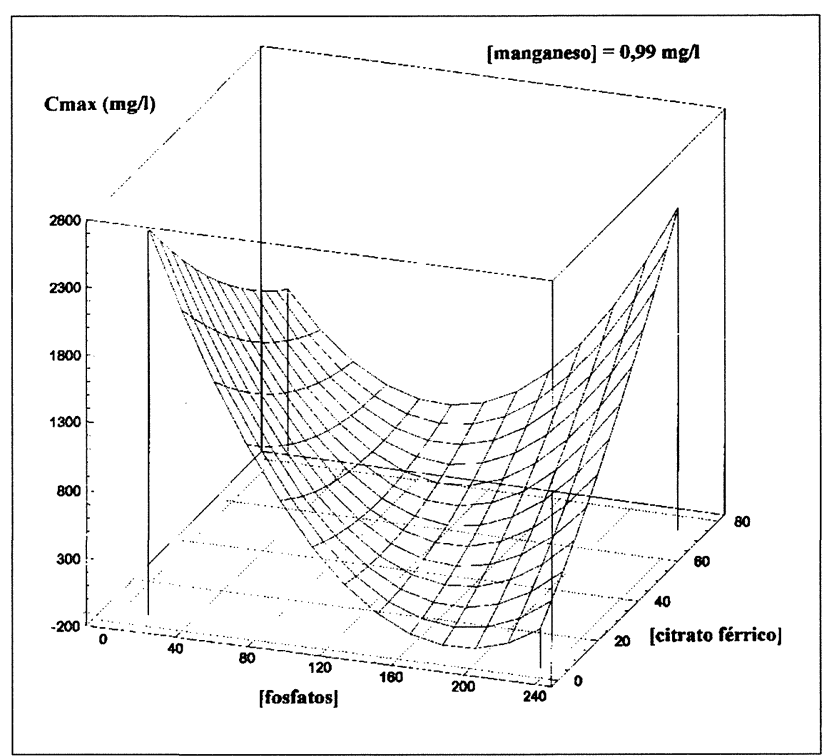

Figura 4

Superficie de respuesta estimada para la concentración máxima de biomasa alcanzada por el cultivo (Cmax) (mg/l) vs concentraciones de citrato férrico y fosfatos en el medio, para la segunda serie experimental.

La concentración de cloruro de manganeso se fija en $0,99 \mathrm{mg} / \mathrm{l}$. Las unidades de los factores se expresan en $\mathrm{mg} / \mathrm{l}$.

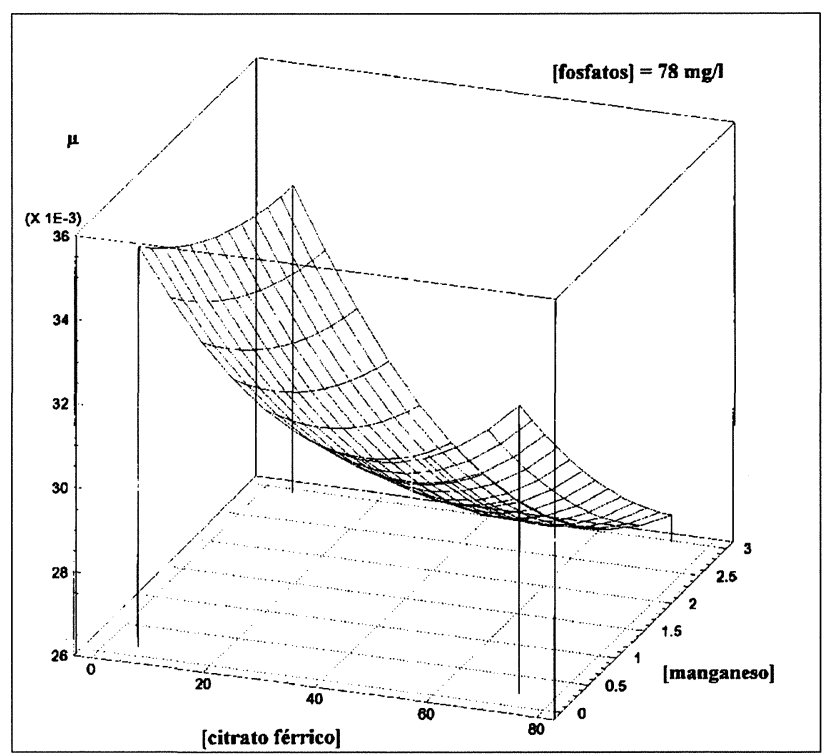

Figura 5

Superficie de respuesta estimada para la velocidad específica de crecimiento $(\mu)\left(h^{-1}\right)$ vs concentraciones de cloruro de manganeso y citrato férrico, para la segunda serie experimental.

La concentración de fosfatos se fija en $78 \mathrm{mg} / \mathrm{l}$.

Las unidades de los factores se expresan en $\mathrm{mg} / \mathrm{l}$. 
$\mu_{\max }(\mu$ máxima) para esta microalga, pese a tener un coeficiente de determinación $(0,7349)$ menor que en la primera serie experimental, pero con el mismo nivel de significación. Este hecho apoya la hipótesis de la existencia de una limitación en la primera serie que hemos sugerido, y que el valor de $\mu_{\max }$ debe ser el valor obtenido en ausencia de cualquier limitación ambiental.

Pretendemos con este trabajo mostrar la rapidez y eficacia de estos métodos estadísticos de optimización, y su aplicación al estudio del comportamiento de sistemas biológicos con relación a los factores considerados en el mismo; (un modelo empírico que explique en torno al $90 \%$ los resultados experimentales con microorganismos de esta naturaleza, permite inferencias bastante fiables de caracter práctico). No obstante, los diseños deben ser muy definidos en sus variables, y tener un conocimiento muy amplio del problema en cuestión.

Como siempre, queda a criterio del investigador, en función de sus posibilidades tanto de tiempo como de material el hacer uso de estos métodos, tomando siempre en consideración la gran importancia que tiene la etapa del diseño experimental.

\section{BIBLIOGRAFÍA}

1. Fernández-Reiriz, M.J.; Pérez-Camacho, A.; Ferreiro, M.J.; Blanco, J.; Planas, M.; Campos, M.J. y Labarta, U.- "Biomass production and variation in the biochemical profile (total protein, carbohydrates, RNA lipids and fatty acids) of seven species of marine microalgae". Aquaculture 83 (1989) 17-37.

2. Whyte, J.N.- "Biochemical composition and energy content of six species of phytoplankton used in mariculture of bivalves". Aquaculture 75 (1987) 193-302.

3. Dyerberg, J.- "Linoleate-derived polyunsaturated fatty acids and prevention of atherosclerosis".- Nutrition Reviews 44 (1986) 125-134.

4. Iacono, J.M. y Dougherty, R.M.- "Effects of polyunsaturated fats on blood pressure".- Annu. Rev. Nutr. 13 (1993) 243-240.

5. Nelson, M.D.- "Diet therapy in coronary disease. Effect on mortality of high-protein, high-seafood, fat-controlled diet".- Geriatrics 12 (1972) 103-116.

6. Singh, M.D. y Chandra, M.D.- "Biochemical and cellular effects of fish and fish oils".- Progress Food Nutrition Science 12 (1988) 371-419.

7. Innis, S.M.- "Essential fatty acids in growth and development".- Prog Lipid Res. 30 (1991) 39-103.

8. Simopoulos, A.P.- "Omega-3 fatty acids in health and disease and in growth and development".- Am.J. Clin. Nutr. 54 (1991) 438-463.

9. Ben-Amotz, A.; Tornabene, T.G. y Thomas, W.H.- "Chemical profile of selected species of microalgae with emphasis on lipids".- J. Phycol. 21 (1985) 72-81.

10. López Alonso, D.; Molina Grima, E.; Sánchez Pérez, J.A. García Sánchez, J.L. y García Camacho, F.- "Isolation of clones of Isochrysis galbana rich in eicosapentaenoic acid".- Aquaculture 102 (1992a) 363-371.

11. Plakas, S.M. y Guarino, A.M.- "Omega-3 fatty acids and fish oils: Is the news all good?".- Proceedings of Eleventh Annual Tropical and Subtropical Fisheries Conference of the Americas (1986).
12. Molina Grima, E.; Sánchez Pérez, J.A.; García Sánchez, J.L.; García Camacho, F. y López Alonso, D.- "EPA from Isochrysis galbana. Growth conditions and productivity".- Process Biochem. 27 (1992) 299-305.

13. Utting, S.D.- "Influence of nitrogen availability on the biochemical composition of three unicellular marine algae of commercial importance".- Aquacult. Eng. 4 (1985) 175-190.

14. Herzig, R. y Falkowsky, P.G.- "Nitrogen limitation in Isochrysis galbana (Haptophyceae). I. Photosynthetic energy conversion and growth efficiencies".- J. Phycol. 25 (1989) 462-471.

15. Sattur, A.P. y Karanth, N.G.- "Production of microbial lipids: I. Development of a mathematical model".- Biotechnology Bioengineering 34 (1989) 863-867.

16. Cochran, W.G. y Cox, G.M.- "Experimentos factoriales". En "Diseños Experimentales".- Trillas S.A. (ed). México. (1965) 177-214.

17. Peña Sánchez de Rivera, D.- "Modelos polinómicos". En "Estadística. Modelos y métodos. 2. Modelos lineales y series temporales".Alianza Editorial. Madrid. (1992) 510-528.

18. Deming, S.N. y Morgan, S.L.- "System theory". En "Experimental design: a chemometric approach".- Elsevier (ed). Amsterdam. (1987) $1-19$

19. Ukeles, R.- "The effect of temperature on the growth and survival of several marine algae species ".- Biological Bulletin 120 (1961) 255-264.

20. Kaplan, D.; Cohen, Z. y Abeliovich, A.- "Optimal growth conditions for Isochrysis galbana".- Biomass 9 (1986) 37-48.

21. Kain, J.M. y Fogg, G.E.- "Studies on the growth of the marine phytoplankton. II. Isochrysis galbana Parke".- J. Mar. Biol. Ass. U.K. 37 (1958) 781-788.

22. Montgomery, D.C.- "Métodos y diseños de superficie de respuesta". En "Diseño y análisis de experimentos".- Grupo Editorial Iberoamérica S.A. México. (1991) 467-509.

23. Box, G.E.P. y Behnken, D.W.- "Some new three level designs for the study of quantitative variables".- Technometrics 2 (1969) 455-475.

24. Molina Grima, E.; Sánchez Pérez, J.A.; García Camacho, F.; García Sánchez, J.L. y López Alonso, D.- "n-3 PUFA productivity in chemostat cultures of microalgae".- Appl. Microbiol. Biotechnol. 38 (1993b) 599-605.

25. Yongmanitchai, W. y Ward, O.P.- " $\omega-3$ fatty acids. Alternative sources of production".- Process Biochem. 24 (1989) 117-125.

26. López Alonso, D.; Molina Grima, E.; Sánchez Pérez, J.A.; García Sánchez, J.L. y García Camacho, F.- "Fatty acid variation among different isolates of a single strain of Isochrysis galbana". Phytochemistry 31 (1992b) 3901-3904.

27. Sánchez Pérez, J.A.- "Productividad en ácidos grasos poliinsaturados $n-3$ de la microalga marina Isochrysis galbana. Condiciones de crecimiento y selección fenótipica".- Tesis Doctoral. Universidad de Granada. (1992) p 312.

28. Lepage, G. y Roy, C.C.- "Improved recovery of fatty acid through direct transterification without prior extraction of purification".- J. Lipid Res. 25 (1984) 1391-1396.

29. García Camacho, F.; Molina Grima, E.; Martínez Sancho, Mª.E. y Sánchez Villasclaras, S.- "Determinación de ácidos grasos en microalgas marinas. Comparación de diversos métodos de extracción lipídica".- Grasas y Aceites 41 (1990) 13-18.

30. García Sánchez, J.L.; Molina Grima, E.; García Camacho, F.;Sánchez Pérez, J.A. y Giménez Giménez, A.- "Cuantificación de ácidos grasos a partir de biomasa microalgal".- Grasas y Aceites 44 (1993) 348-353.

31. Raven, J.A.- "Predictions of $\mathrm{Mn}$ and $\mathrm{Fe}$ efficiencies of phototrophic growth as a function of light availability for growth and for $\mathrm{C}$ assimilation pathway".- New Phytol. 116 (1990) 1-18.

(Recibido: Marzo 1993) 